$\Rightarrow$

\section{Going places with VHL}

Studies over recent years have identified genes that are thought to contribute only to a metastatic phenotype. However, there are undoubtedly numerous pathways that result in the same phenotype, and the background of initial genetic changes could be influential. Joan Massagué and colleagues have found that epigenetic changes contribute to metastasis in the clear-cell subtype of renal-cell carcinomas (ccRCCs)

alterations in the epigenetic regulation of HIF target genes are associated with metastasis

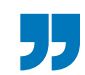
in which the tumour suppressor gene von Hippel-Lindau ( $V H L)$ is inactivated.

In the presence of oxygen, VHL is involved in the degradation of the $\alpha$-subunits of the transcription factor hypoxia inducible factor (HIF), and loss of VHL in ccRCC leads to the stabilization of HIF1a and HIF $2 \alpha$ and the inappropriate transcription of HIF target genes. Although VHL inactivation does

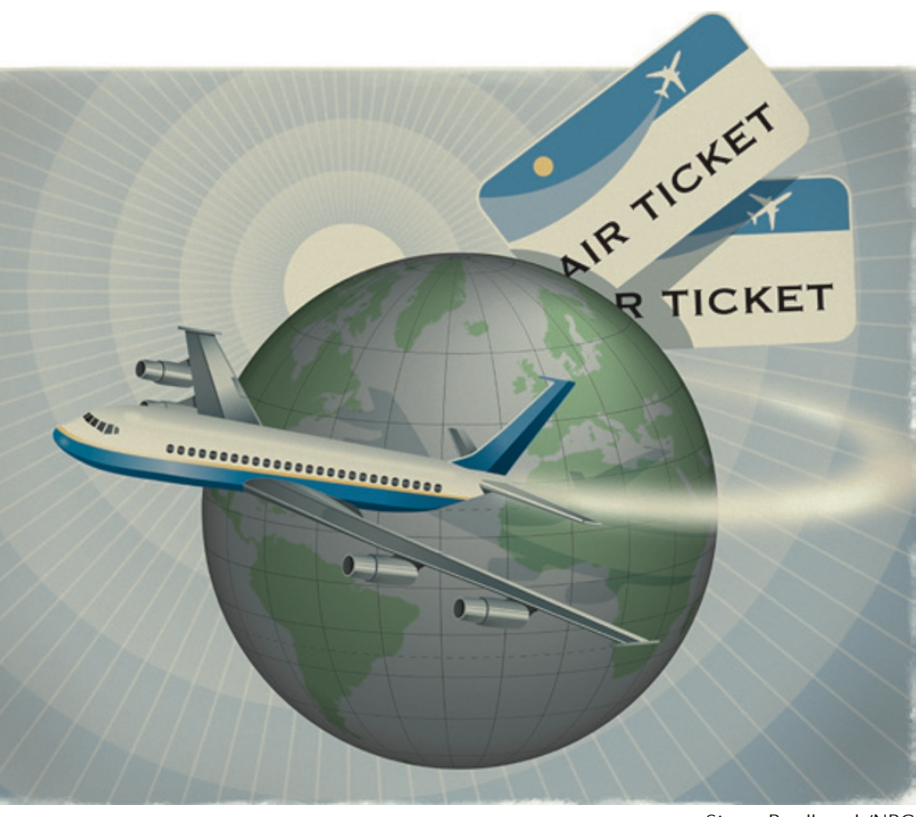

Simon Bradbrook/NPG not correlate with a poor prognosis in ccRCC, the expression of the HIF $2 \alpha$ target gene chemokine (C-X-C motif) receptor 4 (CXCR4) correlates with metastasis in ccRCC. Therefore, Massagué and colleagues examined whether the increased expression of CXCR4 and other potential metastatic genes downstream of the VHL-HIF axis occurs as a result of epigenetic changes.

The authors used a ccRCC cell line established from a patient with metastatic disease and then selected for highly metastatic variants through tail vein injection into immunocompromised mice. Genome-wide transcription profiling identified 155 genes associated with the metastatic phenotype of these cell variants. This gene set was refined to a core set of 50 genes (termed the renal cancer metastasis signature 50 (RMS50)) that are also expressed in ccRCC gene expression profiles that form the GSE2109 data set in the Gene Expression Omnibus. Additional gene expression profiling studies showed that a subset of these genes responded to VHL inactivation and were transcriptional targets of HIF $2 \alpha$. Of these, the authors focused on CXCR4 and cytohesin 1 interacting protein (CYTIP), as their expression was increased across all metastatic ccRCC cell lines that the authors investigated. Moreover, knockdown of CXCR4 or CYTIP reduced lung metastasis in vivo.

Why is expression of these genes increased in metastatic ccRCC cells? Neither gene was amplified, nor was there any difference in mRNA stability or promoter activity. Thus, the authors investigated DNA methylation in the initial parental ccRCC cells and their highly metastatic variants. They found that CYTIP was methylated in the parental cells, but methylation levels were reduced in the metastatic cell lines. In addition, low levels of CYTIP methylation correlated with poor prognosis in patients with ccRCC. Changes in DNA methylation levels were not evident in CXCR4, and instead the authors found that alterations in histone methylation were important. Actively transcribed genes are associated with trimethylation of histone 3 lysine 4 (H3K4me3), whereas inactive genes are associated with trimethylation of $\mathrm{H} 3 \mathrm{~K} 27$, a mark that is produced by members of Polycomb repressor complex 2 (PRC2). The authors showed that $\mathrm{H} 3 \mathrm{~K} 27$ methylation at CXCR4 is reduced in metastatic ccRCC cells. Reduced expression of the PRC2 gene suppressor of zeste 12 homologue (SUZ12) resulted in increased expression of CXCR4, and reduced expression of SUZ12 correlated with metastatic disease. Although the VHL-HIF axis had no effect on the levels of H3K27 methylation, it did influence the levels of H3K4 methylation, indicating that reduced levels of $\mathrm{H} 3 \mathrm{~K} 27 \mathrm{me} 3$ through the reduction of SUZ12 expression results in CXCR4 being marked as an actively transcribed gene in metastatic ccRCC cells.

The authors conclude that alterations in the epigenetic regulation of HIF target genes are associated with metastasis and that the loss of VHL function, which contributes to ccRCC initiation, also contributes to metastasis.

Nicola McCarthy

ORIGINAL RESEARCH PAPER Vanharanta, S. et al. Epigenetic expansion of VHL-HIF signal output drives multiorgan metastasis in renal cancer. Nature Med. 9 Dec 2012 (doi:10.1038/nm.3029) 